\title{
Large-System Phase-Space Dimensionality Loss in Stationary Heat Flows
}

\author{
Harald A. Posch ${ }^{\text {a }}$, William G. Hoover ${ }^{\mathrm{b}}$ \\ ${ }^{\mathrm{a}}$ Institute for Experimental Physics, University of Vienna, Boltzmanngasse 5, \\ A-1090 Vienna, Austria \\ ${ }^{\mathrm{b}}$ Department of Applied Science, University of California at Davis/Livermore and \\ Methods Development Group, Lawrence Livermore National Laboratory, \\ Livermore, California 94551-7808, USA
}

\begin{abstract}
Thermostated tethered harmonic lattices provide good illustrations of the phasespace dimensionality loss $\Delta D$ which occurs in the strange-attractor distributions characterizing stationary nonequilibrium flows. We use time-reversible nonequilibrium molecular dynamics, with two Nosé-Hoover thermostats, one hot and one cold, to study a family of square heat-conducting systems. We find a phase-space dimensionality loss which can exceed the dimensionality associated with the two driving Nosé-Hoover thermostats by as much as a factor of four. We also estimate the dimensionality loss $\Delta D_{\mathcal{H}}$ in the purely Hamiltonian part of phase space. By measuring the projection of the total dimensionality loss there we show that nearly all of the loss occurs in the Hamiltonian part. Thus this loss, which characterizes the extreme rarity of nonequilibrium states, persists in the large-system thermodynamic limit.
\end{abstract}

Key words: Fractals, Heat Flow, Irreversibility, Phase-Space Dimensionality PACS: 05.10.-a, 05.45.Df, 44.10.+i, 05.70.-a

\section{Introduction}

Aoki and Kusnezov stressed the usefulness of the " $\phi$ " model in studying the size-dependence of classical heat conductivity simulations [1]. They used this model to show that the entropy production associated with steady heat flow

Email addresses: posch@ls.exp.univie.ac at (Harald A. Posch), hoover3@llnl.gov (William G. Hoover).

Preprint submitted to Elsevier Science 22 October 2018 
becomes extensive, $(\propto N)$, as the system size $N$ increases. When such heatflow simulations are driven with two time-reversible Nosé-Hoover thermostats $[2,3,4,5,6,7]$ imposing two temperatures, "hot" and "cold", the steady-state phase-space distribution function becomes a multifractal object $[8,9,10]$. The information dimension of this multifractal object lies below the corresponding equilibrium one by an extensive $(\propto N)$ "dimensionality loss" $\Delta D$. The dimensionality loss, like the closely-related thermodynamic entropy production $\dot{S}$, is approximately quadratic in the deviation from equilibrium brought about by the temperature gradient $\nabla T$ :

\section{$\Delta D \propto N(\nabla \ln T)^{2}$}

This quadratic dependence is the usual prediction of linear transport theory. Its evaluation quantifies the rarity of nonequilibrium stationary states.

About ten years ago [11] we studied phase-space dimensionality loss in a family of two-dimensional shear flows. Those simulations were limited to relatively small systems. In that shear-flow work we were able to find systems for which the dimensionality loss barely, but significantly, exceeded the total dimensionality associated with the coordinates, momenta, and friction coefficients $\{q, p, \zeta\}$ of the thermostated boundaries.

At that time we stated that our data definitely showed that nonequilibrium stationary states can exhibit a reduced dimensionality, relative to equilibrium, even when projected into the subspace occupied by purely-Hamiltonian degrees of freedom. This interpretation was controversial [12,13]. Many researchers had difficulty in accepting that nonequilibrium stationary states are typically associated with reduced dimensionality. In order to confirm that our interpretation was correct, we recently studied dimensionality reduction for stationary flows using the (Hamiltonian) $\phi^{4}$ model. Because heat transfer using this model requires only the simplest of boundaries - a single hot degree of freedom and a single cold one, in the simplest one-dimensional case -it turned out to be easy to get relatively large dimensionality losses [2].

A simple but convincing two-dimensional case involves the heat transfer from a single hot particle (with five phase-space coordinates $\left\{x_{H}, y_{H}, p_{x H}, p_{y H}, \zeta_{H}\right\}$ ) to a single cold one (with its own five coordinates) through a medium of $N-2=23$ Hamiltonian particles (with 92 more phase-space coordinates, for a total of $4 N+2=102$ phase-space coordinates). Typical $(x, y)$ particle trajectories in such a 25-particle system are shown in Fig. 1. In the case of $N=16$ particles discussed in Ref. 2 the phase-space strange attractor turned out to have a dimensionality loss of 12.5 , relative to the equilibrium distribution, exceeding the number of thermostated phase-space coordinates (5 hot and 5 cold) by 2.5 .

The present work has two goals, (i) characterizing the overall loss of phase- 


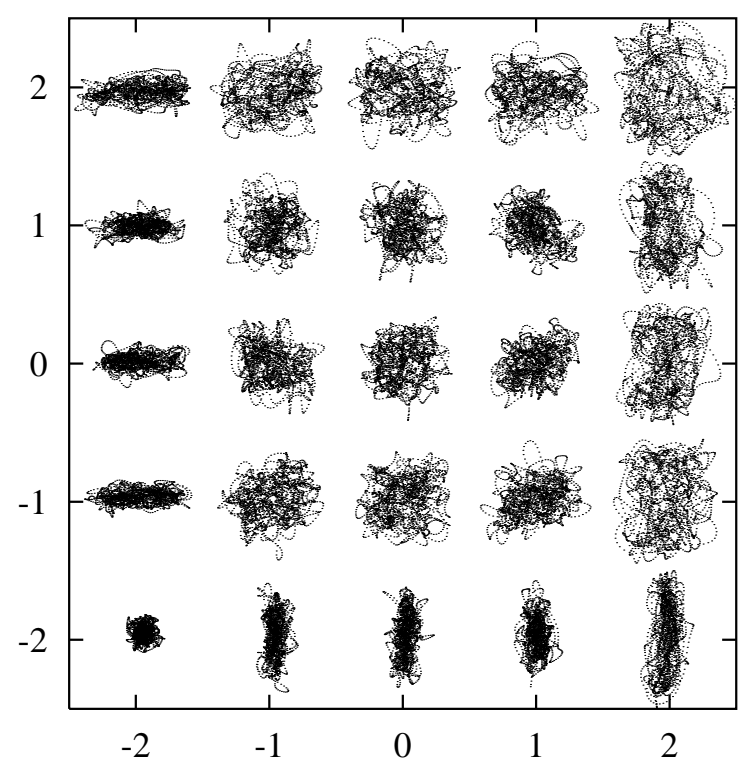

Fig. 1. Geometry of a 25-particle two-dimensional steady-state system with a full-phase space dimensionality reduction $\Delta D=21.6$. The upper righthand particle is "hot" and the lower lefthand one is "cold". The remaining 23 particles obey conventional Hamiltonian mechanics. The trajectories shown were generated with hot and cold temperatures $T_{H}=0.009$ and $T_{C}=0.001$ imposed by two Nosé-Hoover thermostats with relaxation times of $\tau=5$.

space dimensionality $\Delta D$, and (ii) estimating the corresponding loss $\Delta D_{\mathcal{H}}$ in the Hamiltonian unthermostated part of phase space. This two-part study requires the numerical evaluation of the instantaneous Lyapunov exponents $\{\lambda(t)\}$, as well as their associated vectors $\{\delta\}$. The required techniques are sketched in Sec. II. In Sec. III we describe the $\phi^{4}$ model. In Sec. IV we undertake a systematic study of the number-dependence of the phase-space dimensionality loss $\Delta D$. We consider a family of systems of the type illustrated in Fig. 1. The numerical work described in Sec. IV shows convincingly a $\sqrt{1 / N}$ deviation from the large-system limit, with an extrapolated dimensionality loss of as much as four times (40) the number of dimensions (10) associated with the thermal driving mechanism. Next, in Sec. V, we study the projection of this dimensionality loss into the purely Hamiltonian subspace. We find that nearly all of the loss occurs in that subspace despite the purely Hamiltonian form of the equations of motion there. We discuss the projection technique and the importance of rapid rotation in phase space [14] to an understanding of the strange-attractor's dimensionality reduction. The conclusions which follow from this work take up the final section. 


\section{Lyapunov Exponent Calculations}

The usual "Lyapunov exponents", $\left\{\lambda_{j}\right\}$, are time averages, over a sufficiently long time for convergence, of "local" (instantaneous) exponents $\left\{\lambda_{j}(t)\right\}$,

$$
\lambda_{j} \equiv\left\langle\lambda_{j}(t)\right\rangle
$$

Here, and in what follows, we use angular brackets $\langle\ldots\rangle$ to indicate longtime averages. Each of the instantaneous exponents has associated with it an "offset" vector $\delta_{j}$ in phase space which describes the direction in which the growth or decay of phase-space separation is measured. If the offset vectors are infinitesimal in length, as in the present work, they can be replaced by a parallel set of unit vectors in "tangent space".

The time evolution of the offset vector directions is governed by a continuous Gram-Schmidt orthonormalization which forces the vectors to remain mutually perpendicular and to evolve at fixed length. The two types of constraints, orthogonality and fixed length, can be imposed by a triangular array of Lagrange multipliers, $\Lambda_{i j}$, where $1 \leq j \leq i \leq n$ in an $n$-dimensional phase space [15]. When the usual Cartesian coordinates are used the orthonormal offset vetors rotate rapidly [14].

The usual Lyapunov exponents $\left\{\lambda_{j}\right\}$ are the long-time-averaged values of the diagonal Lagrange multipliers $\left\{\Lambda_{j j}\right\}$ required to enforce the orthonormalization constraints:

$$
\lambda_{j}=\left\langle\lambda_{j}(t)\right\rangle \equiv\left\langle\Lambda_{j j}\right\rangle .
$$

At any instant of time the instantaneous Lyapunov exponents $\lambda(t)$ represent the orthogonal measurements of the $n$ growth (or decay) rates determined by the dynamical matrix $D$. $D$, an $n \times n$ matrix, is itself a phase function. The $i$ th row of $D$ is made up of the $n$ derivatives of the $i$ th equation of motion with respect to the $n$ phase variables.

The eigenvalues and eigenvectors of the dynamical matrix are relatively complicated [16]. The eigenvalues are mainly rapidly varying complex-conjugate pairs, with the imaginary parts frequently vanishing, at phase-space singularities corresponding to parallel eigenvectors. Despite this complexity the orthonormal basis provided by the offset vectors makes it possible to measure smooth and well-defined growth rates. The time reversibility of the equations of motion guarantees related reversibility properties for $D$ and its eigenvectors and eigenvalues. Nevertheless, the past-based Lyapunov vectors governed by $D$ show a time-symmetry breaking intimately related to the second law of thermodynamics $[3,8,9,11,14,16]$.

Consider a simple textbook [3](Sec. 5.4) illustration of these ideas, the motion of a driven thermostated particle in one dimension. The motion takes place in 
the three-dimensional $(q, p, \zeta)$ phase space with three Lyapunov exponents:

$$
\begin{aligned}
& \lambda_{1}=\left\langle\lambda_{1}(t)\right\rangle=\left\langle\Lambda_{11}\right\rangle \\
& \lambda_{2}=\left\langle\lambda_{2}(t)\right\rangle=\left\langle\Lambda_{22}\right\rangle \\
& \lambda_{3}=\left\langle\lambda_{3}(t)\right\rangle=\left\langle\Lambda_{33}\right\rangle .
\end{aligned}
$$

The equations of motion (with unit mass, force constant, and relaxation time) are

$$
\dot{q}=p ; \dot{p}=+1-\zeta p ; \dot{\zeta}=p^{2}-1,
$$

for which the long-time solution is an attractor:

$$
\{q(t), p(t), \zeta(t)\} \longrightarrow\{t,+1,+1\}
$$

The matrix of equation-of-motion derivatives $D$ is

$$
D=\left(\begin{array}{ccc}
0 & +1 & 0 \\
0 & -\zeta & -p \\
0 & 2 p & 0
\end{array}\right)
$$

On the attractor the momentum $p$ and friction coefficient $\zeta$ can be replaced by their limiting values, $(+1,+1)$. The three tangent-space $\delta$ vectors follow the ordinary differential equations:

$$
\begin{aligned}
& \dot{\delta}_{1}=D \cdot \delta_{1}-\Lambda_{11} \delta_{1} \\
& \dot{\delta}_{2}=D \cdot \delta_{2}-\Lambda_{21} \delta_{1}-\Lambda_{22} \delta_{2} \\
& \dot{\delta}_{3}=D \cdot \delta_{3}-\Lambda_{31} \delta_{1}-\Lambda_{32} \delta_{2}-\Lambda_{33} \delta_{3}
\end{aligned}
$$

where the six Lagrange multipliers follow easily from the time derivatives of the six orthonormality conditions:

$$
\begin{gathered}
\delta_{1}^{2}=\delta_{2}^{2}=\delta_{3}^{2} \equiv 1 ; \\
\delta_{1} \cdot \delta_{2}=\delta_{2} \cdot \delta_{3}=\delta_{3} \cdot \delta_{1} \equiv 0 .
\end{gathered}
$$

The steady-state time variation of the Lagrange multipliers, and the three $\delta$ vectors are shown in Fig. 2. The three Lyapunov exponents, like their three instantaneous values $\left\{\lambda_{j}(t)=\Lambda_{j j}\right\}$, sum to -1 :

$$
\frac{\partial \dot{q}}{\partial q}+\frac{\partial \dot{p}}{\partial p}+\frac{\partial \dot{\zeta}}{\partial \zeta} \equiv \lambda_{1}(t)+\lambda_{2}(t)+\lambda_{3}(t)=-1
$$

Note that the largest (time-averaged) Lyapunov exponent is 0 in this case, corresponding to a phase-space displacement in the direction of the motion 

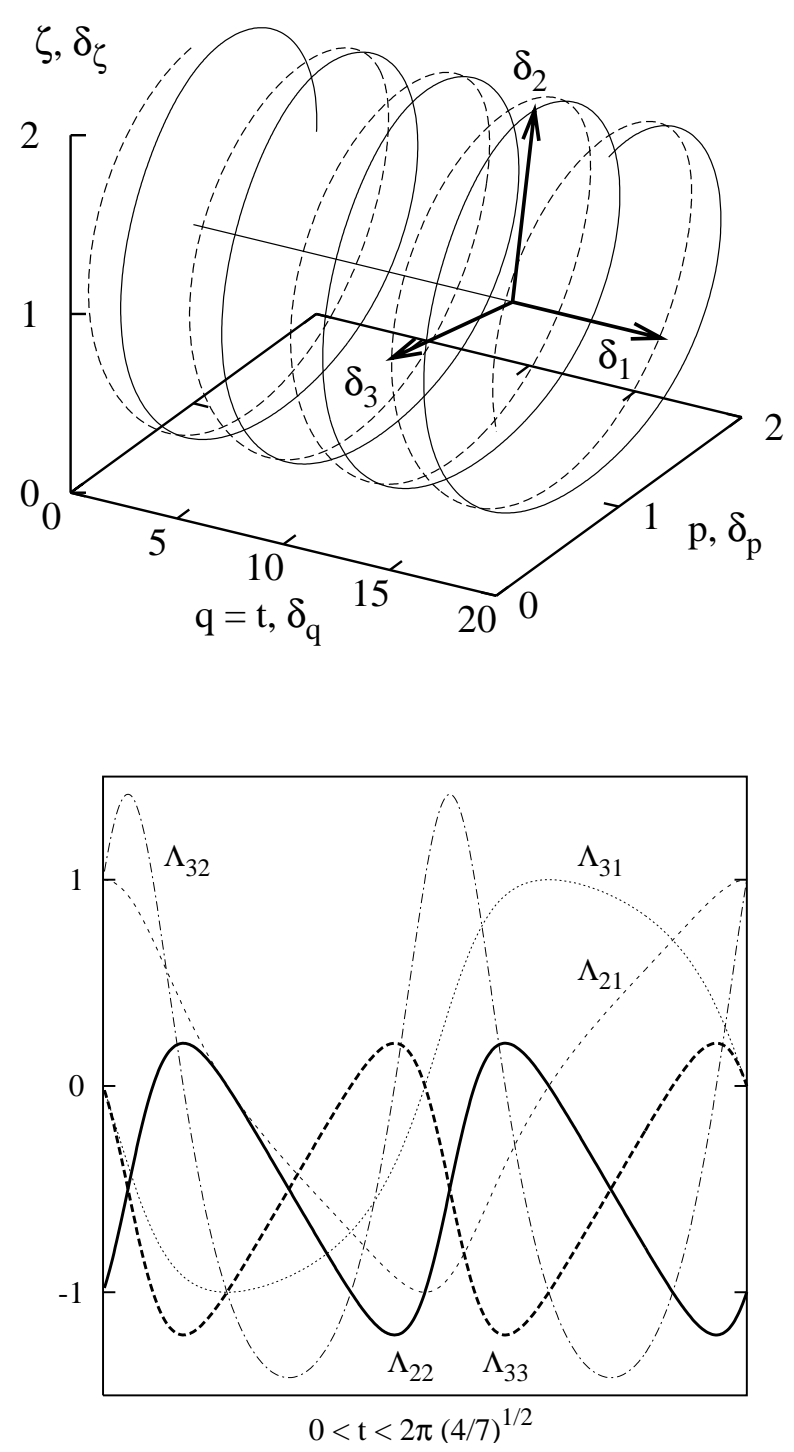

Fig. 2. Bottom: Time dependence of the five nonvanishing Lagrange multipliers for a field-driven thermostated particle in one dimension, as discussed in Sec. II. The time dependence of the vectors $\left\{\delta_{2}, \delta_{3}\right\}$ for this same problem is shown at the top. The period of the oscillation is $2 \pi \sqrt{4 / 7}$ though the phase has no significance in the stationary state.

$\delta_{1}=(1,0,0)$. The remaining five Lagrange multipliers, as well as the vectors $\delta_{2}$ and $\delta_{3}$, oscillate periodically, with a period of $2 \pi \sqrt{4 / 7}$.

An analogous computation for an $n$-dimensional set of vectors involves computational work of order $n^{3}$. Nonequilibrium steady states generate chaotic attractors rather than the simple fixed point of the damped oscillator example. Nevertheless, the basic steps are the same: (i) propagating a phase- 
space reference trajectory; (ii) simultaneously propagating offset vectors in the neighborhood of the reference, with Lagrange multipliers and/or GramSchmidt orthonormalization imposing orthonormality; and (iii) averaging the diagonal multipliers to find the Lyapunov spectrum. Let us now consider the many-body model for which we carry out such investigations.

\section{$3 \quad \phi^{4}$ Heat Flow in Two Dimensions}

In the " $\phi$ " model we use here $[1,2]$ we choose a square nearest-neighbor harmonic lattice with a quadratic Hooke's Law pair potential for neighboring particles $i$ and $j$,

$$
\phi(r)=\frac{\kappa_{2}}{2}(r-d)^{2} ; r=\left|r_{i}-r_{j}\right|>0
$$

In addition, each particle is tethered to its lattice site with a quartic potential, $\frac{\kappa_{4}}{4} \delta r^{4}$. The quartic tethers have two nice consequences. First, they provide "external forces", and so prevent momentum conservation and ballistic energy transport. Second, they can provide chaos, so that the dynamics can have one or more positive Lyapunov exponents. The two consequences together can give Fourier heat conduction, even in one or two space dimensions. The heat conductivity for the $\phi^{4}$ model remains finite in the large-system limit, unlike many models, for which the conductivity vanishes or diverges in the largesystem limit.

Here we simulate nonequilibrium heat-conducting stationary states by imposing thermostating forces on two of the $N$ particles. The thermostating forces are Nosé-Hoover [3,4] feedback forces $\{-\zeta p\}$, linear in both the time-reversible friction coefficients $\{\zeta\}$ and the momenta $\{p\}=\left\{\left(p_{x}, p_{y}\right)\right\}$. The thermostated equations of motion for the hot and cold particles (one of each) are:

$$
\begin{gathered}
\left\{m \dot{x}=p_{x} \quad ; m \dot{y}=p_{y}\right\} \\
\left\{\dot{p}_{x}=F_{x}-\zeta p_{x} ; \dot{p}_{y}=F_{y}-\zeta p_{y}\right\} \\
\dot{\zeta}_{(H \text { or } C)}=\left[\left(p^{2} / 2 m k T_{(H \text { or } C)}\right)-1\right] / \tau^{2},
\end{gathered}
$$

where $\tau$ is the characteristic response time of the thermostat forces, $\{-\zeta p\}$. The full phase space describing this $N$-body two-dimensional system has $4 N+$ 2 dimensions, with the extra two corresponding to the hot and cold friction coefficients $\zeta_{H}$ and $\zeta_{C}$.

For convenience in numerical work we choose the particle mass $m$, the spring constants $\kappa_{2}$ and $\kappa_{4}$, and the nearest-neighbor lattice spacing $d$ all equal to unity. The remaining parameters to set are the hot and cold temperatures, 
which we arbitrarily choose equal to the values from Ref. 2,

$$
k T_{H}=0.009 ; k T_{C}=0.001
$$

and the thermostat relaxation times. We vary these times in the numerical work, but with the simplifying restriction that $\tau_{H}$ and $\tau_{C}$ have a common value, which we denote as $\tau$. We use the classic fourth-order Runge-Kutta integrator throughout, with a timestep $d t=0.001$ or 0.002 . To avoid numerical errors we have generated and compared results from two fully-independent simulation codes, one written in Vienna and the other written in Livermore.

\section{Numerical Results $-\Delta D$}

The numerical evaluation of the dimensionality loss $\Delta D$ is based on the connection between the Lyapunov spectrum and the dimensionality of the phasespace strange attractor. The Lyapunov exponents give the time-averaged relative growth and decay rates of the principal axes of a comoving infinitesimal phase-space hypersphere (or "extension in phase"). Kaplan and Yorke conjectured, evidently correctly [17], that a partial sum of these exponents (beginning with the largest one and proceeding toward the most negative one) changes from positive to negative when the (linearly-interpolated) number of terms in the sum is equal to the dimensionality of the phase-space strange attractor. This conjecture is "almost obvious". It is evident that the (hyper)volume of any phase-space object with a positive sum of time-averaged Lyapunov exponents must diverge. Likewise a negative sum of time-averaged exponents indicates a vanishing hypervolume at long times. Any stationary process must generate an attractor which neither vanishes nor diverges.

The main difficulty in computing Kaplan-Yorke information dimensions is the unfavorable time-dependence associated with constraining the phase-space offset vectors to remain perpendicular to one another. With Gram-Schmidt orthonormalization in an $n$-dimensional phase space $n$ vectors, with $n$ components each, must all be propagated in time for sufficiently long that the time-averaged growth rates have converged. The computational work in orthogonalizing $n(n-1) / 2$ pairs of $n$-dimensional vectors varies as the cube of the number of particles, so that present computer speeds and processor numbers allow us to follow no more than a few hundred particles. In the present work we consider the simplest possible square systems of from $4(2 \times 2)$ to $144(12 \times 12)$ particles. The relaxation time $\tau$ is a free parameter. We choose it in the range $1 \ldots 8$. Results become insensitive to $\tau$ once $\tau$ exceeds 6 , which is comparable to the inverse Debye frequency of the lattice. Representative results for the largest Lyapunov exponent $\lambda_{1}$ and the dimensionality loss from the Kaplan-Yorke conjecture, $\Delta D$, are given in Table 1. 
Table 1

Representative data for the total phase-space dimensionality loss $\Delta D$ and the Hamiltonian projection $\Delta D_{\mathcal{H}}$ as a function of system size and the Nosé-Hoover relaxation time $\tau$. The largest Lyapunov exponent, $\lambda_{1}$ is also tabulated. The two boundary temperatures (imposed by a single hot and a single cold particle) are 0.009 and 0.001 in all cases. The estimated error is \pm 0.4 for $\Delta D$ and $\Delta D_{\mathcal{H}}$, and \pm 0.0005 for $\lambda_{1}$.

\begin{tabular}{ccccc|ccccc}
$\sqrt{N}$ & $\tau$ & $\Delta D$ & $\Delta D_{\mathcal{H}}$ & $\lambda_{1}$ & $\sqrt{N}$ & $\tau$ & $\Delta D$ & $\Delta D_{\mathcal{H}}$ & $\lambda_{1}$ \\
\hline 4 & 1 & 10.3 & 10.6 & 0.0633 & 4 & 4 & 17.6 & 15.6 & 0.0284 \\
5 & 1 & 12.7 & 13.0 & 0.0593 & 5 & 4 & 21.2 & 19.4 & 0.0332 \\
6 & 1 & 14.7 & 15.2 & 0.0560 & 6 & 4 & 23.7 & 22.2 & 0.0359 \\
7 & 1 & 16.4 & 17.0 & 0.0540 & 7 & 4 & 26.7 & 25.3 & 0.0337 \\
8 & 1 & 17.9 & 18.5 & 0.0510 & 8 & 4 & 28.6 & 27.4 & 0.0337 \\
9 & 1 & 19.3 & 19.8 & 0.0496 & 9 & 4 & 29.7 & 28.5 & 0.0344 \\
10 & 1 & 20.2 & 20.7 & 0.0474 & 10 & 4 & 31.1 & 30.0 & 0.0329 \\
12 & 1 & 20.5 & 21.0 & 0.0443 & 12 & 4 & 33.8 & 32.9 & 0.0305 \\
& & & & & & & & & \\
4 & 6 & 18.6 & 16.0 & 0.0275 & 4 & 8 & 18.8 & 15.7 & 0.0273 \\
5 & 6 & 21.9 & 19.6 & 0.0323 & 5 & 8 & 22.1 & 19.3 & 0.0330 \\
6 & 6 & 24.6 & 22.6 & 0.0340 & 6 & 8 & 25.0 & 22.5 & 0.0340 \\
7 & 6 & 27.5 & 25.5 & 0.0333 & 7 & 8 & 27.7 & 25.4 & 0.0339 \\
8 & 6 & 29.5 & 27.6 & 0.0339 & 8 & 8 & 29.5 & 27.4 & 0.0325 \\
9 & 6 & 30.9 & 28.2 & 0.0327 & 9 & 8 & 31.0 & 28.9 & 0.0324 \\
10 & 6 & 32.4 & 30.8 & 0.0320 & 10 & 8 & 32.9 & 30.8 & 0.0321 \\
12 & 6 & 34.2 & 32.7 & 0.0317 & 12 & 8 & 34.5 & 32.7 & 0.0318
\end{tabular}

Our own previous work, on color conductivity and shear flow [11], strongly suggests deviations in the Lyapunov spectrum of order the inverse system width, $\sqrt{1 / N}$ in two dimensions. The present results are roughly consistent with this finding though an even slower variation with $N$ provides a comparable fit. We know of no previous systematic study of the variation of the Lyapunov spectrum with $\tau$. A cursory investigation shows that the dimensionality loss varies roughly as $\tau^{-1}$. Thus the dimensionality loss, with fixed boundary temperatures of 0.009 and 0.001 , can be represented by

$$
\Delta D=42.7(5)-\frac{89(2)}{\sqrt{N}}-\frac{13.2(5)}{\tau}
$$

The standard deviation affecting the last digits of the fit parameters are given in brackets. We compare the second expression with our numerical data in Fig. 3. The rather good fit indicates that the dimensionality loss persists in the large-system limit. We investigate the loss further in the following section. 


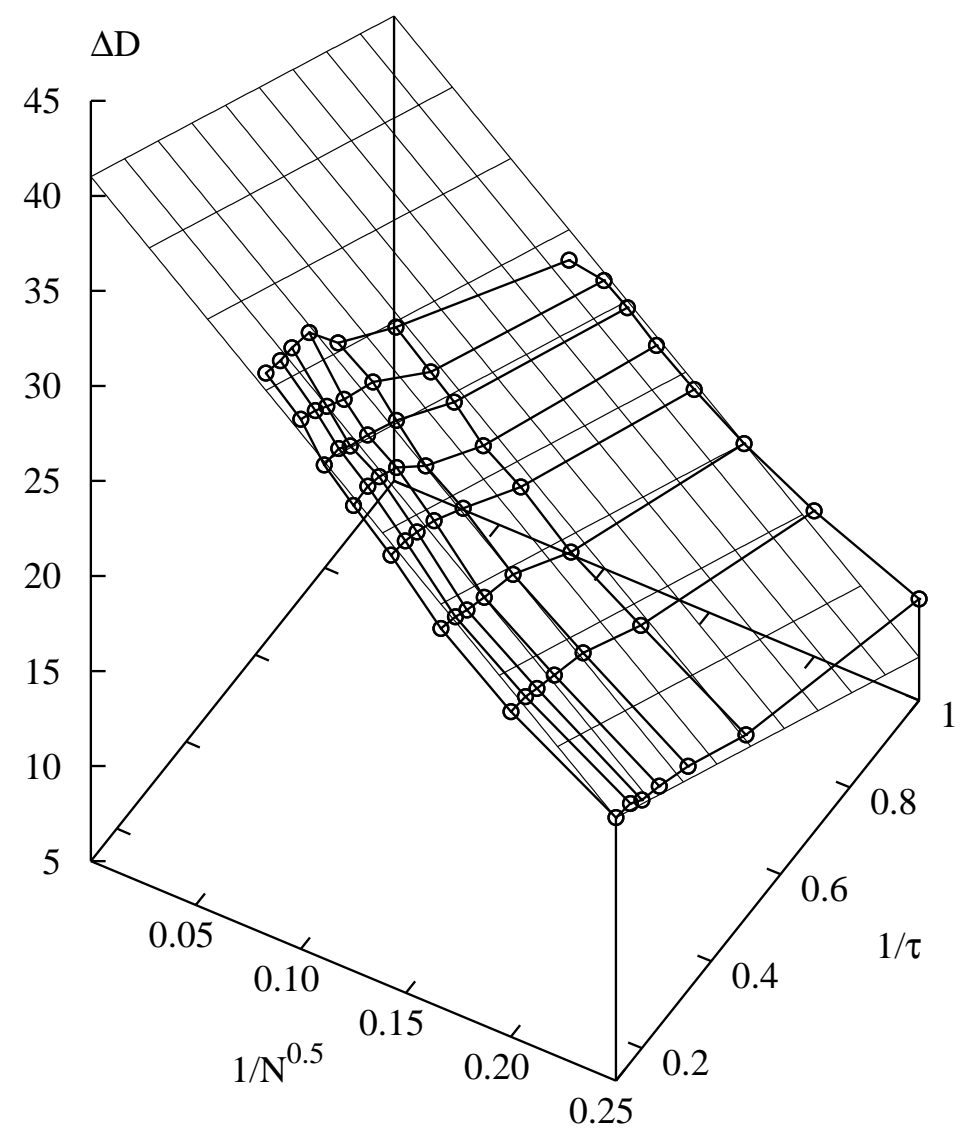

Fig. 3. Comparison of the fit $\Delta D=42.7-\left(89 / N^{1 / 2}\right)-(13.2 / \tau)$ to the simulation results for the dimensionality loss in the full $4 N+2$-dimensional phase space.

\section{$5 \quad$ Hamiltonian Projection: $\Delta D_{\mathcal{H}}$}

Ten years ago [14] we studied the rotation rate of the $n$ phase-space offset vectors $\left\{\delta_{j}\right\}$. We found that the rotation rate increases very rapidly with system size, soon becoming very large relative to the Lyapunov exponents themselves. This observation suggests that the instantaneous growth and decay rates in phase space - the instantaneous Lyapunov exponents - might become isotropic in the large-system limit. This suggests that the measured growth and decay rates are also closely associated with the subspaces spanned by the corresponding phase-space offset vectors $\{\delta\}$. Because the instantaneous Lyapunov exponents measure radial expansion and contraction, without any explicit rotational contribution, the instantaneous growth rates $\left\{\lambda_{j}(t)=\Lambda_{j j}\right\}$ associated with every one of the phase-space directions contributing to a particular vector are identical. The contributions in a fixed phase-space direction are the summed-up contributions from the entire set of Lyapunov vectors.

At a particular phase-space point these contributions of the principal axes 
of the dynamical matrix to the Lyapunov exponents are all proportional to $\cos ^{2}(\theta)$, where $\theta$ is the angular difference between the principal axis and the corresponding instantaneous $\delta$ vector. Because the Lyapunov exponents measure growth or decay in the direction of $\delta$, the same logarithmic growth rate, $(d / d t) \ln \delta$, applies to each component of the vector.

In the full phase space the information dimension of the strange attractor is given by the number of Lyapunov exponents whose sum is zero:

$$
\sum_{j=1}^{k} \lambda_{j}=0 \longrightarrow D=k ; \Delta D=4 N+2-k \text {. }
$$

It has to be emphasized that $k$ (as well as $k_{\mathcal{H}}$ introduced below) is not an integer. A linear interpolation between two successive values of the Lyapunov sum is implied, with $k$ chosen such that the interpolated sum is precisely zero. In the Hamiltonian subspace the Lyapunov exponents contribute according to their projections into that space:

$$
\begin{gathered}
\sum_{j=1}^{k_{\mathcal{H}}}\left\langle\cos ^{2}\left(\theta_{j}\right) \lambda_{j}(t)\right\rangle=0 \longrightarrow D_{\mathcal{H}}=\sum_{j=1}^{k_{\mathcal{H}}}\left\langle\cos ^{2}\left(\theta_{j}\right)\right\rangle ; \\
\Delta D_{\mathcal{H}}=\sum_{j=k_{\mathcal{H}}}^{4 N+2}\left\langle\cos ^{2}\left(\theta_{j}\right)\right\rangle=4 N-8-\sum_{j=1}^{k_{\mathcal{H}}}\left\langle\cos ^{2}\left(\theta_{j}\right)\right\rangle .
\end{gathered}
$$

The $\cos ^{2}(\theta)$ form of the projection is required by the condition that changing the sign of the offset vectors leaves the growth rate unchanged. This form follows from the quadratic form describing an infinitesimal phase-space hyperellipsoid centered on a moving trajectory point. Note also that this weighting satisfies the normalization of the projections:

$$
\sum_{j=1}^{4 N+2} \cos ^{2}\left(\theta_{j}\right) \equiv 4 N-8
$$

Partial sums give an effective number of exponents in the Hamiltonian projection of the full phase space. Thus the analog of Kaplan and Yorke's conjecture for the Hamiltonian subspace is the effective number of exponents, $\sum\left\langle\cos ^{2}(\theta)\right\rangle$, at which the projected sum, $\sum\left\langle\lambda(t) \cos ^{2}(\theta)\right\rangle$, vanishes.

We explored the Kaplan-Yorke analog for the present problem, computing the delta vectors and their projections. The results are interesting. First, we noted that the summed-up local Lyapunov exponents are not strongly correlated with the directions in the subspace:

$$
\sum \lambda\left\langle\cos ^{2}(\theta)\right\rangle \simeq \sum\left\langle\lambda(t) \cos ^{2}(\theta)\right\rangle
$$

This suggests that all the time-averaged projections of the various vectors into 


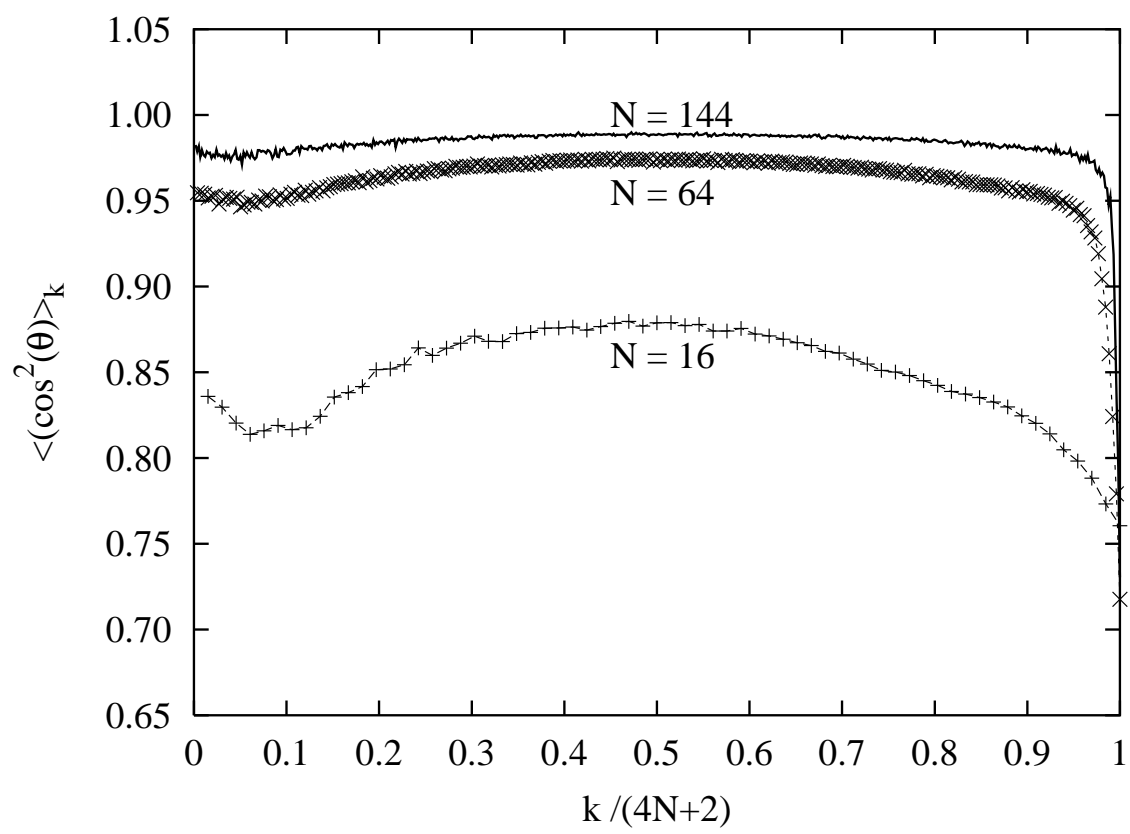

Fig. 4. Time-averaged projections $\left\{\left\langle\cos ^{2}(\theta)\right\rangle_{k}\right\}$ of the full-space $\left\{\delta_{k}\right\}$ into the Hamiltonian portion of phase space for $4 \times 4,8 \times 8$, and $12 \times 12$ particles. The Nosé-Hoover time $\tau$ is 6 . It is evident that in the large-system limit the influence of the boundary degrees of freedom disappears.

the Hamiltonian subspace are similar,

$$
\left\langle\cos ^{2}(\theta)\right\rangle \simeq(4 N-8) /(4 N+2) .
$$

See Figs. 4 and 5. Fig. 4 demonstrates that the projection of the vectors becomes increasingly uniform as system size is increased, and is quite close to the average value, $(4 N-8) /(4 N+2)$ expected for fully random projection directions. Fig. 5 compares the two estimates for the projected Lyapunov sums,

$$
\sum \lambda\left\langle\cos ^{2}(\theta)\right\rangle \text { and } \sum\left\langle\lambda(t) \cos ^{2}(\theta)\right\rangle
$$

The two sums vanish at nearly the same projected dimensionality, $\sum \cos ^{2}(\theta)$, indicating that the correlation of the exponents with direction is small.

We have used the analog of the Kaplan-Yorke formula to estimate the dimensionality reduction in the Hamiltonian subspace, $\Delta D_{\mathcal{H}}$, and show these results in Fig. 6. Just as in the full phase space, the loss of dimensionality varies smoothly with relaxation time and system size, and may be represented by

$$
\Delta D_{\mathcal{H}}=41.2(5)-\frac{94(3)}{\sqrt{N}}-\frac{10.1(5)}{\tau}
$$

It is worth pointing out that a naïve approach to dimensionality loss in the Hamiltonian subspace could be based on an orthonormalization of the Hamil- 


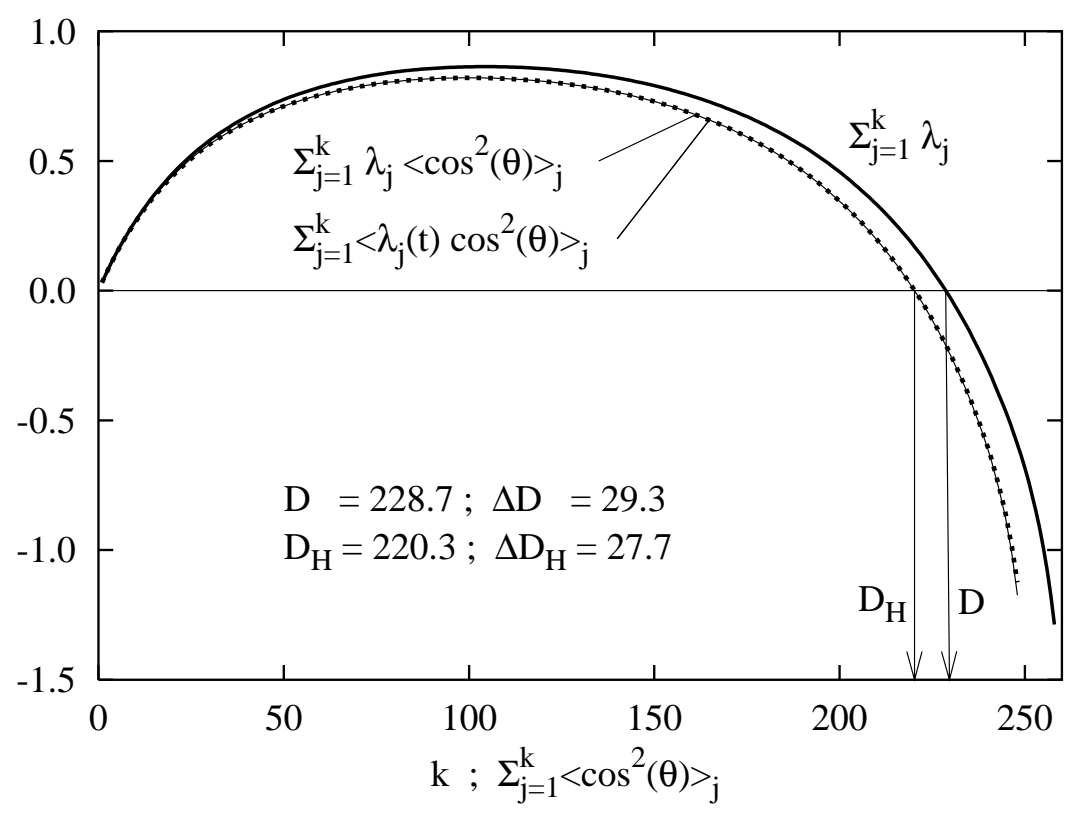

Fig. 5. Typical summed Lyapunov spectra for the full space and for two estimates of the Hamiltonian projection. The data shown here, for 64 particles and for a thermostat response time $\tau=5$, show partial sums of the 258 Lyapunov exponents, $\sum_{j \leq k} \lambda_{j}$, and the two projections, $\sum_{j \leq k} \lambda_{j}\left\langle\cos ^{2}\left(\theta_{j}\right)\right\rangle ; \sum_{j \leq k}\left\langle\lambda_{j}(t) \cos ^{2}\left(\theta_{j}\right)\right\rangle$, as functions of $k$ and $\sum_{j \leq k}\left\langle\cos ^{2}\left(\theta_{j}\right)\right\rangle$. The two projections are actually different but indistinguishable on this scale. Kaplan and Yorke's form for the information dimension is the linearly-interpolated value of $k$ for which the linearly-interpolated sum vanishes. The analog, for the Hamiltonian portion of phase space, is the interpolated value of $\sum \cos ^{2}(\theta)$ at which the corresponding projected sum of exponents vanishes.

tonian subspace only, propagating $\delta$ vectors with the Hamiltonian equations of motion but using the thermostated equations of motion for the underlying reference trajectory. This approach, which we explored years ago and which has recently been considered independently by Ken Aoki [private communications, 2002], is equivalent to considering the Lyapunov spectrum for a Hamiltonian system subject to time-dependent forces $\{F(t)\}$, where the forces are due to the thermostated particles. Such equations of motion, being Hamiltonian, satisfy Liouville's Theorem $[9,18]$, and guarantee that the corresponding Lyapunov spectrum is made up of pairs of positive and negative exponents, with zero sum.

\section{Conclusions}

The " $\phi$ " model shows conclusively that the nonequilibrium steady-state loss of phase-space dimensionality can easily exceed the dimensionality associated with the system boundaries. The present results also confirm that the de- 


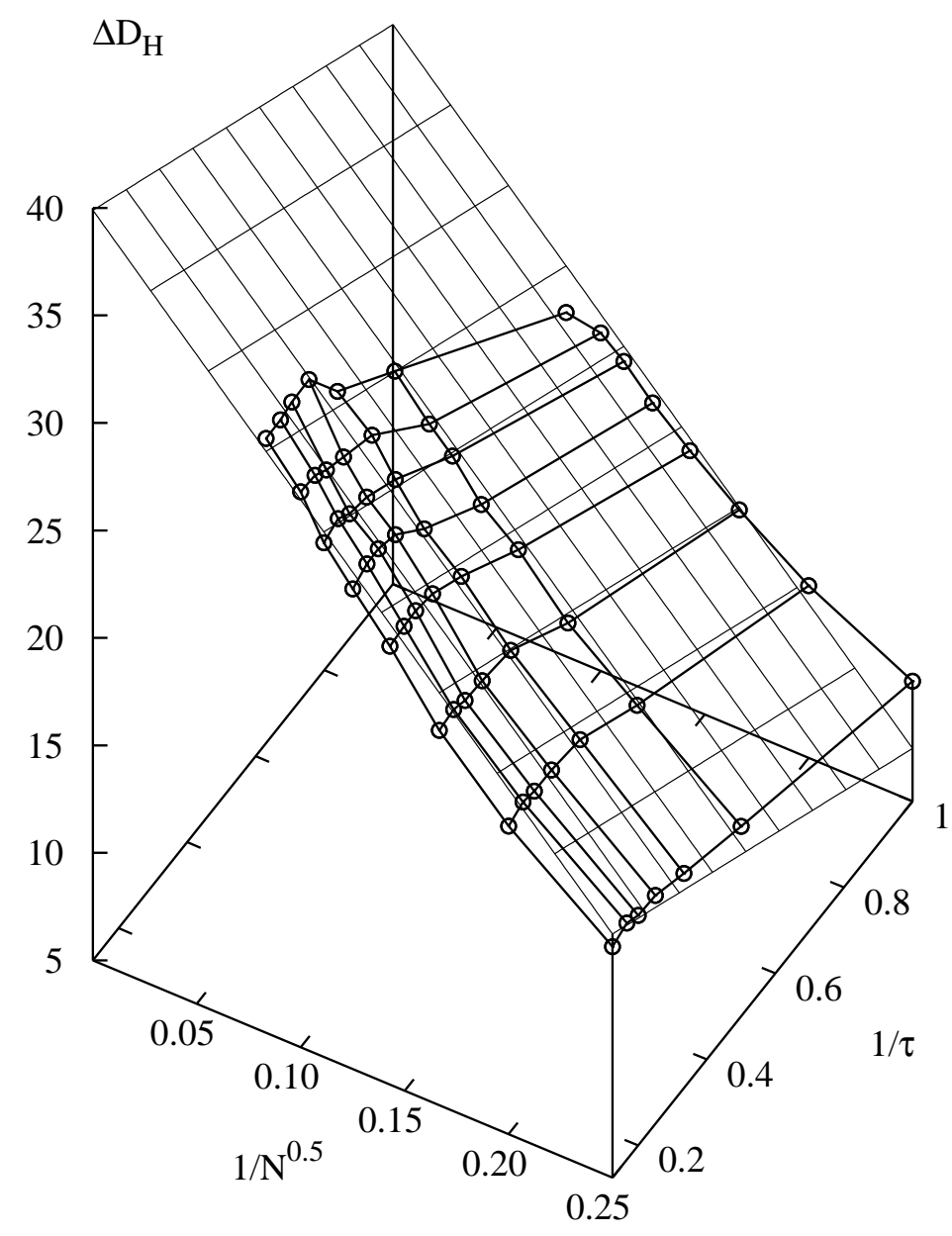

Fig. 6. Comparison of the dimensionality loss $\Delta D_{\mathcal{H}}$ in the Hamiltonian subspace to a fit, $\Delta D_{\mathcal{H}}=41.2-94 / \sqrt{N}-10.1 / \tau$, to the data.

viations from the large-system limit vary according to a power law, $N^{-p}$, $1 / 4 \leq p \leq 1 / 2$, roughly compatible with the inverse of the system size, $1 / \sqrt{N}$ in two dimensions. For $\tau \leq 5$ they have in addition deviations in the relaxation frequency $\propto(1 / \tau)$. The quartic tethers are a particularly useful feature of the model, which make it possible to carry out the simulations without the need to take thermal expansion explicitly into account.

The dimensionality loss $\Delta D$ is expected to be extensive as has been stressed in the introduction. For fixed thermostat temperatures $T_{H}$ and $T_{C}$ the temperature gradient is determined by the system size, proportional to $1 / \sqrt{N}$ in two dimensions, and $\Delta D$ becomes independent of $N$. This is indeed the case in the large-particle limit $N \rightarrow \infty$. Our simulations thus confirm the extensivity of the dimensionality reduction for stationary heat flow in the linear-response limit. Far from equilibrium weak deviations from this $N$-dependence are found.

Table 1 reveals that for short Nosé-Hoover relaxation times the dimensionality 
loss $\Delta D_{\mathcal{H}}$ in the purely Hamiltonian part of phase space may even slightly exceed the dimensionality loss $\Delta D$ in the full phase space. This is because $\Delta D_{\mathcal{H}}$ is overestimated by one due to the lack of energy conservation in the Hamiltonian subspace once the thermostats are added.

The projection of the phase-space offset vectors $\{\delta\}$ into the Hamiltonian subspace developed here shows that most of the dimensionality loss occurs in a part of the system which obeys purely conservative Hamiltonian equations of motion. Rapid rotation is responsible. This rotation nearly eliminates the correlations between phase-space contraction and direction. Evidently phasespace contraction is not only real, but relatively simple, and certainly must persist in the large-system thermodynamic limit. Thus the present results corroborate our interpretation of the second law of thermodynamics for nonequilibrium stationary states [8]. Such states occupy not just a reduced volume in phase space. They are restricted to a subspace of reduced dimensionality, with the dimensionality loss simply related to the rate of external thermodynamic entropy production. Useful models illustrating dimensionality loss for periodic color conducting or shear flows can now be developed as extensions of this idea.

\section{Acknowledgements}

Work at the University of Vienna was supported by the Austrian Fonds zur Förderung der wissenschaftlichen Forschung, Project P-15348. Work at Livermore was performed under the auspices of the United States Department of Energy through University of California Contract W-7405-Eng-48. We very much appreciate the interest and encouragement of Kenichiro Aoki, Aurel Bulgac, Carol Hoover, Dimitri Kusnezov, and David Ruelle.

\section{References}

[1] K. Aoki and D. Kusnezov, "Lyapunov Exponents, Transport, and the Extensivity of Dimensionality Loss", nlin.CD/0204015.

[2] Wm. G. Hoover, H. A. Posch, K. Aoki, and D. Kusnezov, "Remarks on NonHamiltonian Statistical Mechanics: Lyapunov Exponents and Phase-Space Dimensionality Loss", Europhysics Lett. (to appear, 2002).

[3] Wm. G. Hoover, Time Reversibility, Computer Simulation, and Chaos (World Scientific, Singapore, 1999).

[4] S. Nosé, "A Unified Formulation of the Constant Temperature Molecular Dynamics Methods", J. Chem. Phys. 81, 511-519 (1984). 
[5] D. Kusnezov, A. Bulgac, and W. Bauer, "Canonical Ensembles from Chaos", Annals of Phys. 204, 155-185 (1990); 214, 180-218 (1992).

[6] Wm. G. Hoover and B. L. Holian, "Kinetic Moments Method for the Canonical Ensemble Distribution", Phys. Lett. A 211, 253-257 (1996).

[7] D. J. Evans and B. L. Holian, "The Nosé-Hoover Thermostat", J. Chem. Phys. 83, 4069-4074 (1985).

[8] B. L. Holian, W. G. Hoover, and H. A. Posch, "Resolution of Loschmidt's Paradox: the Origin of Irreversible Behavior in Atomistic Dynamics", Phys. Rev. Lett. 59, 10 (1987).

[9] Wm. G. Hoover, "Liouville's Theorems, Gibbs' Entropy, and Multifractal Distributions for Nonequilibrium Steady States", J. Chem. Phys. 109, 41644170 (1998).

[10] D. Ruelle, "Smooth Dynamics and New Theoretical Ideas in Nonequilibrium Statistical Mechanics", J. Stat. Phys. 95, 393-468 (1999).

[11] H. A. Posch and W. G. Hoover, Nonequilibrium Molecular Dynamics of Classical Fluids", in Molecular Liquids: New Perspectives in Physics and Chemistry, J. J. C. Teixeira-Dias, ed. (Kluwer, Amsterdam, 1992).

[12] M. Mareschal and B. L. Holian, eds., Microscopic Simulations of Complex Hydrodynamic Phenomena, NATO ASI Series in Physics 292 (Plenum, New York, 1994).

[13] Wm. G. Hoover, D. J. Evans, H. A. Posch, B. L. Holian, and G. P. Morriss, Phys. Rev. Letts. 80, 4103 (1998).

[14] H. A. Posch, W. G. Hoover, and B. L. Holian, "Time-Reversible Molecular Motion and Macroscopic Irreversibility", Ber. Bunsenges. Phys. Chem. 94, 250256 (1990).

[15] H. A. Posch and W. G. Hoover, "Lyapunov Instability of Dense Lennard-Jones Fluids", Phys. Rev. A, 38, 473-482 (1988).

[16] Wm. G. Hoover, C. G. Hoover, and H. A. Posch, "Dynamical Instabilities, Manifolds, and Local Lyapunov Spectra Far From Equilibrium", Computational Methods in Science and Technology (Poznań) 7, 55-65 (2001).

[17] J. D. Farmer, E. Ott, and J. A. Yorke, "The Dimension of Chaotic Attractors", Physica 7D, 153-180 (1983).

[18] J. Ramshaw, "Remarks on Non-Hamiltonian Statistical Mechanics", Europhys. Lett. 59, 319-323 (2002). 